\title{
The strong Novikov conjecture for low degree cohomology
}

\author{
Bernhard Hanke • Thomas Schick
}

Received: 25 October 2007 / Accepted: 29 April 2008 / Published online: 23 May 2008

(C) The Author(s) 2008

\begin{abstract}
We show that for each discrete group $\Gamma$, the rational assembly map

$$
K_{*}(B \Gamma) \otimes \mathbb{Q} \rightarrow K_{*}\left(C_{\max }^{*} \Gamma\right) \otimes \mathbb{Q}
$$

is injective on classes dual to $\Lambda^{*} \subset H^{*}(B \Gamma ; \mathbb{Q})$, where $\Lambda^{*}$ is the subring generated by cohomology classes of degree at most 2 (and where the pairing uses the Chern character). Our result implies homotopy invariance of higher signatures associated to classes in $\Lambda^{*}$. This consequence was first established by Connes-Gromov-Moscovici (Geom. Funct. Anal. 3(1): 1-78, 1993) and Mathai (Geom. Dedicata 99: 1-15, 2003). Note, however that the above injectivity statement does not follow from their methods. Our approach is based on the construction of flat twisting bundles out of sequences of almost flat bundles as first described in our work (Hanke and Schick, J. Differential Geom. 74: 293-320, 2006). In contrast to the argument in Connes-Gromov-Moscovici (Geom. Funct.Anal. 3(1): 1-78, 1993), our approach is independent of (and indeed gives a new proof of) the result of Hilsum-Skandalis (J. Reine Angew. Math. 423: 73-99, 1999) on the homotopy invariance of the index of the signature operator twisted with bundles of small curvature.
\end{abstract}

Keywords Novikov conjecture - Almost flat bundles · K-homology

Mathematics Subject Classification (2000) $\quad 19 K 56$

\section{Introduction}

Throughout this paper, we use complex $K$-theory. Let $\Gamma$ be a discrete group and denote by $C_{\max }^{*} \Gamma$ the maximal group $C^{*}$-algebra of $\Gamma$. Recall the following form of the strong Novikov conjecture.

\section{B. Hanke}

Ludwig-Maximilians-Universität München, Munich, Germany

e-mail: hanke@mathematik.uni-muenchen.de

T. Schick $(\varangle)$

Georg-August-Universität Göttingen, Gottingen, Germany

e-mail: schick@uni-math.gwdg.de 
Conjecture 1.1 The Baum-Connes assembly map

$$
A: K_{*}(B \Gamma) \rightarrow K_{*}\left(C_{\max }^{*} \Gamma\right)
$$

is injective after tensoring with the rational numbers.

The Chern character

$$
\operatorname{ch}: K(-) \rightarrow H(-; \mathbb{Q})
$$

is a natural transformation (of $\mathbb{Z} / 2$-graded multiplicative (co-)homology theories) from $K$-homology to rational singular homology (both theories being defined in the homotopy theoretic sense). Let

$$
\Lambda^{*}(\Gamma) \subset H^{*}(B \Gamma ; \mathbb{Q})
$$

be the subring generated by classes of degree at most 2 .

In this paper we verify the strong Novikov conjecture for classes dual to elements in $\Lambda^{*}(\Gamma)$.

Theorem 1.2 Let $h \in K_{*}(B \Gamma)$ be a $K$-homology class such that the map

$$
\Lambda^{*}(\Gamma) \rightarrow \mathbb{Q}, \gamma \mapsto\langle\gamma, \operatorname{ch}(h)\rangle,
$$

given by the Kronecker pairing is nonzero. Then

$$
A(h) \neq 0 \text {. }
$$

As a corollary we obtain the following result on homotopy invariance of higher signatures $(\mathcal{L}(M)$ denotes the $L$-polynomial of $M)$.

Corollary 1.3 [cf. [4], [9]] Let $M$ be a closed connected oriented smooth manifold, let $\Gamma$ be a discrete group and let $f: M \rightarrow B \Gamma$ be a continuous map. Then for all $c \in \Lambda^{*}(\Gamma)$, the higher signature

$$
\left\langle\mathcal{L}(M) \cup f^{*}(c),[M]\right\rangle
$$

is an oriented homotopy invariant.

The discussion of this result in [9] is based on a theorem of Hilsum-Skandalis [6] saying that the index of the signature operator twisted with Hilbert $A$-module bundles ( $A$ being a $C^{*}$-algebra) of small curvature is an oriented homotopy invariant. For flat twisting bundles this result was known before (see e.g. $[7,8,10]$ ). Our proof of Theorem 1.2 is independent of [6]. Indeed, we will illustrate in the last section of this paper how our methods allow the reduction of the Hilsum-Skandalis theorem to the case of flat twisting bundles. Moreover, it should be stressed that the methods of [4] and [9] do just yield the assertion of Corollary 1.3 but not the injectivity statement of Theorem 1.2.

\section{Proof of the main theorem}

By a standard suspension argument we may assume that $h \in K_{0}(B \Gamma)$. Because each discrete group is the direct limit of finitely presented groups and because the classifying space construction, the formation of $C_{\max }^{*}$ and the $K$-theory functors commute with direct limits, it is enough to treat the case of finitely presented $\Gamma$. 
Due to the geometric description of $K$-homology by Baum-Douglas [2], elaborated in [3], there is triple $(M, E, \phi)$, where $M$ is an even dimensional closed connected spin-manifold, $E \rightarrow M$ is a virtual (i.e. $\mathbb{Z} / 2$-graded) hermitian complex vector bundle of finite dimension and $\phi: M \rightarrow B \Gamma$ is a continuous map, such that

$$
\phi_{*}\left(E \cap[M]_{K}\right)=h .
$$

(Strictly speaking, [3] only provides a $\operatorname{spin}^{c}$-manifold; it is an exercise to obtain a spin manifold such that the associated $\operatorname{spin}^{c}$-structure represents the right $K$-homology class). In the formula, we consider $E$ as a class in $K^{0}(M)$, and $[M]_{K}$ is the $K$-theoretic orientation class of the spin manifold $M$.

As $\Gamma$ is finitely presented, we can and will assume that the map $\phi: M \rightarrow B \pi_{1}(M)$ induces an isomorphism of fundamental groups.

Now chose $h \in K_{0}(B \Gamma)$ as in the main theorem and let $(M, E, \phi)$ be a triple representing $h$. Let

$$
v=E \Gamma \times_{\Gamma} C_{\max }^{*} \Gamma
$$

be the canonical flat $C_{\max }^{*} \Gamma$-module bundle over $B \Gamma$.

Denoting by $S^{ \pm} \rightarrow M$ the bundles of positive or negative complex spinors on $M$, respectively, the assembly map is described as follows.

Proposition 2.1 The element

$$
A(h) \in K_{0}\left(C_{\max }^{*} \Gamma\right)
$$

is equal to the Mishchenko-Fomenko index of

$$
D_{E \otimes \phi^{*}(v)}: \Gamma\left((S \otimes E)^{+} \otimes \phi^{*}(v)\right) \rightarrow \Gamma\left((S \otimes E)^{-} \otimes \phi^{*}(v)\right),
$$

the Dirac operator on $M$ twisted with the virtual bundle $E \otimes \phi^{*}(v)$. Here, $E$ is equipped with an arbitrary hermitian connection.

This description of the assembly map will be used in order to show that $A(h) \neq 0$.

For that purpose, let $c \in \Lambda^{*}(\Gamma) \subset H^{*}(B \Gamma, \mathbb{Q})$ be such that

$$
\langle c, \operatorname{ch}(h)\rangle \neq 0 \in \mathbb{Q} .
$$

We can assume that $c \in H^{*}(B \Gamma ; \mathbb{Z})$. In order to keep the exposition transparent, let us first assume that $c \in H^{2}(B \Gamma ; \mathbb{Z})$.

Let $L \rightarrow B \Gamma$ be a complex hermitian line bundle classified by $c$. We pick a unitary connection on the pull back bundle $L^{\prime}=\phi^{*}(L) \rightarrow M$ and denote by $\omega \in \Omega^{2}(M ; i \mathbb{R})$ its curvature form. Let $\pi: \widetilde{M} \rightarrow M$ be the universal cover. Because the universal cover of $B \Gamma$ is contractible, the bundle $\pi^{*}\left(L^{\prime}\right) \rightarrow \widetilde{M}$ is trivial. Fix a unitary trivialization $\pi^{*}\left(L^{\prime}\right) \cong \widetilde{M} \times \mathbb{C}$. With respect to this trivialization, the induced connection on $\pi^{*}\left(L^{\prime}\right)$ is given by a 1-form $\eta \in \Omega^{1}(\widetilde{M} ; i \mathbb{R})$.

Because $U(1)$ is abelian, the curvature form of this connection is equal to $d \eta$ which in turn coincides with $\pi^{*}(\omega)$ by naturality. However, contrary to the form $\pi^{*}(\omega)$, the connection form $\eta$ is in general not invariant under the deck transformation group (this would imply that $\omega$ represents the zero class in $\left.H^{2}(M)\right)$.

We will use the bundle $\pi^{*}\left(L^{\prime}\right)$ in order to construct a flat $A$-module bundle $W \rightarrow M$ with an appropriate $C^{*}$-algebra $A$ along the lines of [5]. The flat bundle $W$ will induce a holonomy representation

$$
C_{\max }^{*} \pi_{1}(M) \rightarrow A
$$


whose induced map in $K$-theory will be used to detect non-triviality of the element $A(h)$ appearing in the main theorem.

The details are as follows. For $t \in[0,1]$ we consider the connection on $\widetilde{M} \times \mathbb{C}$ associated to the 1 -form $t \cdot \eta$. The corresponding curvature form is equal to $t \cdot \pi^{*}(\omega)$ and this is invariant under deck transformations (note that this is in general not true for the forms $t \cdot \eta$ if $t \neq 0$ ).

We would like to use the bundle $L^{\prime}$ to construct a family of almost flat bundles $\left(P_{t}\right)_{t \in[0,1]}$ (cf. [5], Sect. 2) so that [5, Theorem 2.1] can be applied in order to obtain an "infinite product bundle"

$$
V=\prod_{n \in \mathbb{N}} P_{1 / n} \rightarrow M
$$

whose quotient by the corresponding infinite sum bundle will be the desired flat bundle $W \rightarrow M$, [cf. 5, Proposition 3.4]. However, by Chern-Weil theory it is in general impossible to produce a finite dimensional bundle on $M$ whose curvature form is equal to $t \cdot \omega, 0<t<1$ (the associated Chern class would not be integral).

We bypass this difficulty by allowing infinite dimensional bundles. Consider the Hilbert space bundle

$$
\mu=\widetilde{M} \times_{\Gamma} l^{2}(\Gamma) \rightarrow M .
$$

Here, $\Gamma$ acts on the left of $l^{2}(\Gamma)$ by the formula

$$
(\gamma \psi)(x):=\psi\left(x \cdot \gamma^{-1}\right), \quad x, \gamma \in \Gamma .
$$

The forms $t \eta \in \Omega^{1}(\widetilde{M})$ induce a family of connections $\nabla^{t}$ on $\mu$, the connection $\nabla^{t}$ being induced by the $\Gamma$-invariant connection on $\widetilde{M} \times l^{2}(\Gamma)$ which on the subbundle

$$
\widetilde{M} \times \mathbb{C} \cdot 1_{\gamma} \subset \widetilde{M} \times l^{2}(\Gamma)
$$

(identified canonically with $\widetilde{M} \times \mathbb{C}$ ) coincides with $\left(\gamma^{-1}\right)^{*}(t \eta)$.

We wish to regard the bundles $\left(\mu, \nabla^{t}\right)$ as twisting bundles for the Dirac operator on $M$. The index of this Dirac operator will live in the $K$-theory of an appropriate $C^{*}$-algebra $A_{t}$ that we think of as the holonomy algebra of $\mu$ with respect to the connection $\nabla^{t}$.

To define these algebras, let us choose a base point $p \in M$ and a point $q \in \widetilde{M}$ above $p$. We identify the fibre over $p$ with the Hilbert space $l^{2}(\Gamma)$.

Now let

$$
A_{t} \subset B\left(l^{2}(\Gamma)\right)
$$

be the norm-linear closure of all maps $l^{2}(\Gamma) \rightarrow l^{2}(\Gamma)$ that arise from parallel transport with respect to $\nabla^{t}$ along closed curves in $M$ based at $p$. Further, we define a bundle $P_{t} \rightarrow M$ whose fibre over $x \in M$ is given by the norm-linear closure (in $\operatorname{Hom}\left(\mu_{p}, \mu_{x}\right)$ ) of all isomorphisms $\mu_{p} \rightarrow \mu_{x}$ arising from parallel transport with respect to $\nabla^{t}$ along smooth curves connecting $p$ with $x$. In this way we obtain, for each $t \in[0,1]$, a locally trivial bundle $P_{t}$ consisting of free $A_{t}$-modules of rank 1 (the $A_{t}$-module structure given by precomposition) and equipped with $A_{t}$-linear connections. For the notions relevant in this context, we refer the reader e.g. to [11]. Parallel transport on $P_{t}$ along a curve connecting $x$ with $x^{\prime}$ is induced by parallel transport $\mu_{x} \rightarrow \mu_{x^{\prime}}$ with respect to $\nabla^{t}$. The bundle $\mu$ may be recovered from the "principal bundle" $P_{t}$ as an associated bundle, i.e. $\mu=P_{t} \times_{A_{t}} l^{2}(\Gamma)$.

The next lemma is crucial for the calculations which will follow.

Lemma 2.2 Each of the algebras $A_{t}$ carries a canonical trace

$$
\tau_{t}: A_{t} \rightarrow \mathbb{C}
$$


given by

$$
\tau_{t}(\psi):=\left\langle\psi\left(1_{e}\right), 1_{e}\right\rangle
$$

where $1_{e} \in l^{2}(\Gamma)$ is the characteristic function of the neutral element and $\langle-,-\rangle$ is the inner product on $l^{2}(\Gamma)$.

Proof Let $\gamma$ and $\gamma^{\prime}$ be two closed smooth curves based at $p \in M$ and let $\phi_{\gamma}$ and $\phi_{\gamma^{\prime}}$ be parallel transport along $\gamma$ and $\gamma^{\prime}$. We show that

$$
\tau_{t}\left(\phi_{\gamma} \cdot \phi_{\gamma^{\prime}}\right)=\tau_{t}\left(\phi_{\gamma^{\prime}} \cdot \phi_{\gamma}\right) .
$$

We will assume from now on that $\gamma$ and $\gamma^{\prime}$ represent elements in $\pi_{1}(M, p)$ that are inverse to each other (otherwise, both sides of the above equation are zero). We lift the composed curves $\gamma \cdot \gamma^{\prime}$ and $\gamma^{\prime} \cdot \gamma$ to curves $\chi$ and $\chi^{\prime}$ starting at $q$ in the universal cover $\widetilde{M}$. By assumption, both $\chi$ and $\chi^{\prime}$ are closed curves. We need to compare parallel transport of the element

$$
(q, 1) \in \widetilde{M} \times \mathbb{C}
$$

along $\chi$ and $\chi^{\prime}$. Denoting the result of parallel transport along these curves by $\left(q, \xi_{\chi}\right)$ and $\left(q, \xi_{\chi^{\prime}}\right)$, respectively, we have

$$
\xi_{\chi}=\exp \left(\int_{[0,1]}-t \eta_{\chi}(\tau)(\dot{\chi}(\tau)) \mathrm{d} \tau\right)
$$

with the exponential map of the Lie group $S^{1}$. By use of Stokes' formula, the last expression is equal to

$$
\exp \left(\int_{D}-\mathrm{d}(t \eta) \mathrm{d} x\right)=\exp \left(\int_{D}-t \pi^{*}(\omega) \mathrm{d} x\right)
$$

where $D: D^{2} \rightarrow \widetilde{M}$ is a disk with boundary $\chi$ (recall that $\widetilde{M}$ is simply connected). However, the curve $\chi^{\prime}$ is obtained (up to reparametrization) from $\chi$ by applying the deck transformation corresponding to $\gamma^{\prime}$. This implies_-grace to the invariance of the form $\pi^{*}(\omega)$ under deck transformation-that the last expression is equal to $\xi_{\chi^{\prime}}$. Using continuity and linearity of $\tau_{t}$, this shows our assertion.

We now equip $E$ with a unitary connection and consider the twisted Dirac operator

$$
D_{E \otimes P_{t}}: \Gamma\left((S \otimes E)^{+} \otimes P_{t}\right) \rightarrow \Gamma\left((S \otimes E)^{-} \otimes P_{t}\right) .
$$

The index $\operatorname{ind}\left(D_{E \otimes P_{t}}\right) \in K_{0}\left(A_{t}\right)$ satisfies (up to sign) the equation

$$
\tau_{t}\left(\operatorname{ind}\left(D_{E \otimes P_{t}}\right)\right)=\left\langle\operatorname{ch}(\mathcal{A}(M)) \cup \operatorname{ch}(E) \cup \operatorname{ch}_{\tau_{t}}\left(P_{t}\right),[M]\right\rangle
$$

by the Mishchenko-Fomenko index theorem (see [11, Theorem 6.9]). Because the Poincaré dual of $\operatorname{ch}(\mathcal{A}(M))$ equals $\operatorname{ch}\left([M]_{K}\right)$, the last expression is $\left\langle\operatorname{ch}_{\tau_{t}}\left(P_{t}\right), \operatorname{ch}\left([E] \cap[M]_{K}\right)\right\rangle$. Recall that the choice of $(M, E, \phi)$ implies $\phi_{*} \operatorname{ch}\left([E] \cap[M]_{K}\right)=\operatorname{ch}(h)$. The construction of the connection $\nabla^{t}$ and the definition of $\mathrm{ch}_{\tau_{t}}$ (see [11, Definition 5.1]) show

$$
\operatorname{ch}_{\tau_{t}}\left(P_{t}\right)=\exp \left(t \phi^{*} c\right) \in H^{*}(M ; \mathbb{R})
$$

so that finally

$$
\tau_{t}\left(\operatorname{ind}\left(D_{E \otimes P_{t}}\right)\right)=\left\langle\exp \left(t \phi^{*} c\right), \operatorname{ch}\left([E] \cap[M]_{K}\right)\right\rangle=\langle\exp (t c), \operatorname{ch}(h)\rangle \in \mathbb{R}[t],
$$

a polynomial which is different from zero by the assumption $\langle c, \operatorname{ch}(h)\rangle \neq 0$. 
We wish to use this calculation in order to detect non-triviality of $A(h) \in K_{0}\left(C_{\max }^{*} \Gamma\right)$. This is done by constructing a flat bundle on $M$ out of the sequence of bundles $\left(P_{1 / n}\right)_{n \in \mathbb{N}}$ with connections. This sequence is almost flat in the sense of [5, Sect. 2]. Therefore, applying [5, Theorem 2.1.] we obtain a smooth "infinite product bundle"

$$
V=\prod_{n \in \mathbb{N}} P_{1 / n} \rightarrow M
$$

equipped with a connection that we may think of as the product of the connections on $P_{1 / n}$. After passing to the quotient by the infinite sum of the bundles $P_{1 / n}$, we obtain a smooth bundle

$$
W=\left(\prod P_{1 / n}\right) /\left(\bigoplus P_{1 / n}\right) \rightarrow M
$$

of free modules of rank 1 over the $C^{*}$-algebra

$$
A=\left(\prod A_{1 / n}\right) /\left(\overline{\bigoplus A_{1 / n}}\right)
$$

which carries an induced flat connection. For more details of this discussion, we refer the reader to [5], especially to Sect. 2 and the statements before Proposition 3.4.

The flat bundle $W$ induces a unitary holonomy representation of $\pi_{1}(M)$. Because $\phi$ induces an isomorphism $\pi_{1}(M) \cong \Gamma$ by the choice of the triple $(M, \phi, E)$, we hence obtain a $C^{*}$ algebra map

$$
\psi: C_{\max }^{*} \Gamma \rightarrow A
$$

using the universal property of $C_{\max }^{*}$. The induced map in $K$-theory can be analyzed in terms of the $K K$-theoretic description of the index map (cf. [5, Lemma 3.1]). One concludes that $\psi_{*}(A(h)) \in K_{0}(A)$ is equal to the index of the twisted Dirac operator

$$
D_{E \otimes W}: \Gamma\left((S \otimes E)^{+} \otimes W\right) \rightarrow \Gamma\left((S \otimes E)^{-} \otimes W\right)
$$

and this in turn equals the image of the index of the twisted Dirac operator

$$
D_{E \otimes V}: \Gamma\left((S \otimes E)^{+} \otimes V\right) \rightarrow \Gamma\left((S \otimes E)^{-} \otimes V\right)
$$

under the canonical map $p_{*}: K_{0}\left(\prod A_{1 / n}\right) \rightarrow K_{0}(A)$ induced by the canonical projection $p: \prod A_{1 / n} \rightarrow A$.

We have a short exact sequence

$$
K_{0}\left(\bigoplus A_{1 / n}\right) \rightarrow K_{0}\left(\prod A_{1 / n}\right) \stackrel{p_{*}}{\rightarrow} K_{0}(A)
$$

where the left hand group is canonically isomorphic to the algebraic direct sum $\oplus_{n \in \mathbb{N}} K_{0}$ $\left(A_{1 / n}\right)$. Furthermore, the traces $\tau_{1 / n}: A_{1 / n} \rightarrow \mathbb{C}$ all have norm 1 and hence induce a trace

$$
\prod A_{1 / n} \rightarrow \prod \mathbb{C}
$$

Using the canonical isomorphism $K_{0}\left(\oplus A_{1 / n}\right) \cong \oplus K_{0}\left(A_{1 / n}\right)$ [1], we finally get a trace map

$$
\tau: \operatorname{im} p_{*} \rightarrow\left(\prod \mathbb{C}\right) /(\bigoplus \mathbb{C})
$$

Note that the direct sum on the right is understood in the algebraic sense: Any element has only finitely many components different from 0 . Assuming ind $\left(D_{E \otimes W}\right)=0$ we conclude that the element in $\prod \mathbb{C} \oplus \mathbb{C}$ represented by

$$
\left(\operatorname{ind}\left(D_{E \otimes P_{1 / n}}\right)\right)_{n \in \mathbb{N}}
$$


is equal to zero. Combining this with our previous calculation, this means that the polynomial

$$
\langle\exp (t c), \operatorname{ch}(h)\rangle \in \mathbb{R}[t]
$$

is equal to zero for all but finitely many values $t=1 / n, n \in \mathbb{N}$. This implies that this polynomial is identically zero and hence in particular

$$
\langle c, \operatorname{ch}(h)\rangle=0
$$

in contradiction to our assumption. The proof of the main theorem is therefore complete, if $c \in H^{2}(B \Gamma ; \mathbb{Z})$.

We will now discuss the case of general $c \in \Lambda^{*}(\Gamma)$ (still assuming $h \in K_{0}(B \Gamma)$ ). If

$$
c=c_{1} \cup \cdots \cup c_{k}
$$

is a product of classes in $H^{2}(B \Gamma ; \mathbb{Z})$, we replace the bundle $L$ in the above argument by the tensor product bundle

$$
L:=L_{1} \otimes L_{2} \otimes \cdots \otimes L_{k} \rightarrow B \Gamma
$$

where the line bundle $L_{i} \rightarrow B \Gamma$ is classified by $c_{i}$. In an analogous fashion as before, we get bundles

$$
P_{t_{1}, \ldots, t_{k}} \rightarrow M
$$

of Hilbert- $A_{t_{1}, t_{2}, \ldots, t_{k}}$-modules working with the connection

$$
t_{1} \eta(1)+t_{2} \eta(2)+\cdots+t_{k} \eta(k)
$$

on the bundle $\widetilde{M} \times \mathbb{C}$ where $\eta(i)$ is a connection induced from $L_{i}$ and where each $t_{i} \in[0,1]$.

Assuming that $A(h)=0$ we can conclude in this case that the polynomial

$$
\left\langle\exp \left(t_{1} c_{1}\right) \cdots \exp \left(t_{k} c_{k}\right), \operatorname{ch}(h)\right\rangle \in \mathbb{R}\left[t_{1}, \ldots, t_{k}\right]
$$

is equal to zero for all but finitely many

$$
\left(t_{1}, \ldots, t_{k}\right)=\left(1 / n_{1}, \ldots, 1 / n_{k}\right)
$$

where $n_{i} \in \mathbb{N}$. Hence this polynomial is identically 0 and in particular

$$
\left\langle c_{1} \cup \cdots \cup c_{k}, \operatorname{ch}(h)\right\rangle=0
$$

again contradicting our assumption. In the most general case, $c$ being a sum

$$
c=c(1)+\cdots+c(k)
$$

where each $c(i)$ is a product of two dimensional classes in $H^{2}(B \Gamma ; \mathbb{Z})$, the assumption

$$
\langle c, \operatorname{ch}(h)\rangle \neq 0
$$

implies that already for one summand $c(i)$, we have $\langle c(i), \operatorname{ch}(h)\rangle \neq 0$ so that we are reduced to the previous case.

Because we already used a suspension argument in order to restrict attention to classes in $K_{0}(B \Gamma)$, this finishes the proof of the main theorem. 


\section{Hilsum-Skandalis revisited}

If $M$ is an oriented Riemannian manifold of even dimension, recall that $d+d^{*}$, the sum of the exterior differential and its formal adjoint, defines the signature operator

$$
D^{\text {sign }}: \Gamma\left(\Lambda_{+}^{*}(M)\right) \rightarrow \Gamma\left(\Lambda_{-}^{*}(M)\right),
$$

where the \pm -signs indicate \pm 1 -eigenspaces of the Hodge star operator.

If $E \rightarrow M$ is a Hilbert $A$-module bundle over $M$, where $A$ is some $C^{*}$-algebra, we obtain the twisted signature operator

$$
D_{E}^{\text {sign }}: \Gamma\left(\Lambda_{+}^{*}(M) \otimes E\right) \rightarrow \Gamma\left(\Lambda_{-}^{*}(M) \otimes E\right)
$$

which has an index ind $D_{E}^{\text {sign }} \in K_{0}(A)$. The following theorem says that this class is an oriented homotopy invariant, if $E$ has small curvature.

Theorem 3.1 Hilsum-Skandalis [6] Let $M$ and $M^{\prime}$ be closed oriented Riemannian manifolds of the same dimension and let $h: M^{\prime} \rightarrow M$ be an orientation preserving homotopy equivalence. Then there exists a constant $c>0$ with the following property: If $E \rightarrow M$ is a Hilbert A-module bundle with connection $\nabla$ so that the associated curvature form $\Omega_{\nabla} \in \Omega^{2}(M$; End $E)$ satisfies the bound

$$
\left\|\Omega_{\nabla}\right\|<c,
$$

(the norm being defined by the maximum norm on the unit sphere bundle in $\Lambda^{2} M$ and the operator norm on each fibre $\left.\operatorname{End}\left(E_{x}, E_{x}\right)\right)$, then we have

$$
\operatorname{ind}\left(D_{f *(E)}^{\text {sign }}\right)=\operatorname{ind}\left(D_{E}^{\text {sign }}\right) \text {. }
$$

If $E$ is flat, i.e. $\Omega_{\nabla}=0$, this result was proved in [7,8,10]. In this section, we will explain briefly how this special case implies the general statement of Theorem 3.1. Our argument is again based on the construction of a flat bundle out of a sequence of almost flat bundles [5].

Assuming that no $c$ with the stated property exists, we find a sequence of $C^{*}$-algebras $A_{n}$ and a sequence of Hilbert $A_{n}$-module bundles $E_{n} \rightarrow M$ with connections $\nabla_{n}$ so that $\left\|\Omega_{\nabla_{n}}\right\|<\frac{1}{n}$, but

$$
\operatorname{ind}\left(D_{f^{*}\left(E_{n}\right)}^{\text {sign }}\right) \neq \operatorname{ind}\left(D_{E_{n}}^{\text {sign }}\right)
$$

for all $n$. We obtain an almost flat sequence

$$
f^{*}\left(E_{n}\right) \cup E_{n} \rightarrow M^{\prime} \cup M
$$

of Hilbert $A_{n}$-module bundles in the sense of [5, Sect. 2] over the disjoint union $M^{\prime} \cup M$. Applying again the methods in [5], we obtain a flat Hilbert $A$-module bundle

$$
W=\left(\prod f^{*}\left(E_{n}\right) \cup \prod E_{n}\right) /\left(\bigoplus f^{*}\left(E_{n}\right) \cup \bigoplus E_{n}\right) \rightarrow M^{\prime} \cup M
$$

where

$$
A=\left(\prod A_{n}\right) /\left(\overline{\bigoplus A_{n}}\right)
$$

The index $\operatorname{ind}\left(D_{W}^{\text {sign }}\right)$ of the signature operator on $\left(-M^{\prime}\right) \cup M$ (the minus-sign indicates reversal of orientation) twisted by $W$, vanishes by the results in $[7,8,10]$. 
This conclusion leads to the following contradiction. We have a canonical isomorphism

$$
K_{0}\left(\bigoplus A_{n}\right) \cong \bigoplus K_{0}\left(A_{n}\right)
$$

and-assuming that each $A_{n}$ is unital and stable, the last property easily being achieved by replacing each $A_{n}$ by $A_{n} \otimes \mathbb{K}\left(l^{2}(\mathbb{N})\right)$ - a canonical isomorphism

$$
K_{0}\left(\prod A_{n}\right) \cong \prod K_{0}\left(A_{n}\right),
$$

compare the proof of [5, Proposition 3.6]. The signature operator on $\left(-M^{\prime}\right) \cup M$ twisted with the non-flat Hilbert $\prod A_{n}$-module bundle

$$
V:=\prod f^{*}\left(E_{n}\right) \cup \prod E_{n}
$$

has an index

$$
\operatorname{ind}\left(D_{V}^{\text {sign }}\right) \in K_{0}\left(\prod A_{n}\right) \cong \prod K_{0}\left(A_{n}\right)
$$

which is different from 0 for infinitely many factors by our assumption on the bundles $E_{n}$. On the other hand, under the canonical map

$$
p_{*}: K_{0}\left(\prod A_{n}\right) \rightarrow K_{0}(A)
$$

this index maps to the index of the signature operator twisted with the bundle $W$ and this index was identified as 0 . Now the contradiction arises from the fact that by the long exact $K$-theory sequence, the kernel of $p_{*}$ is equal to $K_{0}\left(\oplus A_{n}\right)=\oplus K_{0}\left(A_{n}\right)$ (algebraic direct sum) and therefore $\operatorname{ind}\left(D_{V}^{\text {sign }}\right)$ is not contained in it.

Acknowledgements Bernhard Hanke and Thomas Schick are members of the DFG emphasis programme "Globale Differentialgeometrie" whose support is gratefully acknowledged.

Open Access This article is distributed under the terms of the Creative Commons Attribution Noncommercial License which permits any noncommercial use, distribution, and reproduction in any medium, provided the original author(s) and source are credited.

\section{References}

1. Blackadar, B.: $K$-Theory for Operator Algebras, 2nd edn. Cambridge University Press, Cambridge (1998)

2. Baum, P., Douglas, R.G.: $K$-homology and index theory. In: Operator Algebras and Applications, Part I (Kingston, Ont., 1980), pp. 117-173. Proc. Sympos. Pure Math., 38, Amer. Math. Soc., Providence, R.I.

3. Baum, P., Higson, N., Schick, T.: On the equivalence of geometric and analytic K-homology. Pure Appl. Math. Quart. 3, 1-24, (2007)

4. Connes, A., Gromov, M., Moscovici, H.: Group cohomology with Lipschitz control and higher signatures. Geom. Funct. Anal. 3(1), 1-78 (1993)

5. Hanke, B., Schick, T.: Enlargeability and index theory. J. Differential Geom. 74, 293-320 (2006)

6. Hilsum, M., Skandalis, G.: Invariance par homotopie de la signature à coefficients dans un fibré presque plat. J. Reine Angew. Math. 423, 73-99 (1992)

7. Kaminker, J., Miller, J.G.: Homotopy invariance of the analytic signature operators over $C^{*}$-algebras. J. Oper. Theory 14, 113-127 (1985)

8. Kasparov, G.: Topological invariance of elliptic operators I: $K$-homology, Izv. Akad. Nauk. S.S.S.R. Ser. Mat. 39, 796-838 (1975); English transl. in Math. U.S.S.R. Izv. 9, 751-792 (1975).

9. Mathai, V.: The Novikov conjecture for low degree cohomology classes. Geom. Dedicata 99, 1-15 (2003)

10. Mishchenko, A.: $C^{*}$-algebras and $K$-theory. Lect. Notes Math. 763, 262-274 (1979)

11. Schick, T.: $L^{2}$-index theorems, $K K$-theory and connections. New York J. Math. 11, 387-443 (2005) 